\title{
Evaluation of glutathione S-transferase polymorphism in Iranian patients with type 2 diabetic microangiopathy
}

\author{
Mohammad Bagher Hashemi-Soteh ${ }^{1}$ (D), Amir Ahmadzadeh Amiri² (D, Majid Reza Sheikh Rezaee ${ }^{3}$ (D) \\ Ali Ahmadzadeh Amiri ${ }^{2}$ (D) Rohodin Ahrari ${ }^{4}$, Ahmad Ahmadzadeh Amiri ${ }^{* *}$ and Fatemeh Daneshvar ${ }^{6}$ (D)
}

\begin{abstract}
Background: Overproduction of reactive oxygen species as a result of hyperglycemia in diabetes mellitus leads to microvascular complications. Glutathione S-transferases play important detoxifying roles with antioxidant potentials. This study aimed to assess whether the glutathione S-transferase M1 and T1 genotypes were associated with type 2 diabetes mellitus microangiopathic complications in the Iranian population.

Results: In this case-control study, the frequencies of null GSTM1 and GSTT1 genotypes were 4/72 (5.56\%) and 12/ 72 (16.67\%) respectively, in uncomplicated DM group. The frequencies of null GSTM1 and GSTT1 genotype in complicated DM group were 16/134 (11.94\%) and 37/134 (27.61\%), respectively. The proportion of GSTM1 null genotypes was higher in diabetic nephropathy compared to non-nephropathy $(19.3 \%$ vs. $6.04 \%, P=0.006)$. At GSTT1 locus, patients with diabetic peripheral neuropathy had a higher frequency of deletion compared to those of without neuropathy (30.39\% vs. 23.49\%) ( $P=0.02)$.

Conclusion: Selective polymorphisms encoding GSTM1 and GSTT1genes may prove useful as genetic markers to recognize individuals with an increased trend in developing diabetic nephropathy and neuropathy, respectively. This will help better identify individuals at higher risk toward microvascular complications of type 2 diabetes due to genetic susceptibility.
\end{abstract}

Keywords: Glutathione S-transferases, Gene polymorphisms, Diabetic retinopathy, Diabetic nephropathy, Diabetic neuropathy

\section{Background}

Diabetes mellitus (DM) is a chronic metabolic disease; leaving the patient in need of continuous medical consideration with health plans to reduce multi-organ complications [1]. Diabetic microangiopathy is specified as a microvascular disorder with long-term complications including diabetic neuropathy, retinopathy, and nephropathy [2]. Hyperglycemia in type 2 diabetes (T2D) may lead to overproduction of reactive oxygen species (ROS) in the cells which in turn activates protein kinase (PKC),

\footnotetext{
* Correspondence: ahmadzdh@yahoo.com

${ }^{5}$ Diabetes Research Center, Mazandaran University of Medical Sciences, Sari, Iran

Full list of author information is available at the end of the article
}

resulting in a raise in advanced glycation end products (AGEs) [3]. Microvascular angiopathy in T2D may occur from high endogenous inflammatory factors such as plasma interleukin 6 and tumor necrosis factor-alpha [4]. In addition, low antioxidant capacity increases the susceptibility toward diabetes complications $[5,6]$ Glutathione S-transferase (GST) is one of the most important detoxifying enzymes [7]. Several cytosolic and membrane-bound enzymes have been detected in human species that are further subdivided into alpha, mu, pi, kappa, sigma, theta, omega, and zeta isoenzymes [8]. GST genes may represent polymorphic traits that lead to changes in enzymes activity [9]. Mu loci have three different alleles, containing gene deletion (GSTM1-0), and 
two indifferent mutations (GSTM1a and GSTM1b) [10, 11]. Two different alleles have been recognized for GSTT1 that give functional specifications to the gene [12] Polymorphisms in the GST gene may disturb defense mechanisms against oxidative stress which eventually lead to diabetic microvascular complications. There are many studies regarding GST polymorphism in various diseases, but only a few have addressed the role of GST polymorphisms in diabetes and its microvascular complications.

Zaki et al. demonstrated an increased risk of developing T2DM but not its vascular-related complications among Egyptian cases having heterozygous GSTP1 polymorphism alone as well as when combined with GSTM1 null genotype [13]. However, Purkait, et al. showed significantly increased frequency of GSTM1 null genotype in the Indian diabetic nephropathy patients [14]. In the current study, we aimed to determine the genotype frequency of the GSTM1, GSTT1 polymorphisms to understand whether or not the GST polymorphisms are associated with a higher risk for microvascular complications related to DM in Iranian population.

\section{Methods}

\section{Study population}

This case-control study was designed based on the Ethical Declaration of Helsinki. The research protocol was approved on 24 September 2014 by the ethical committee under the Ethical ID: IR.MAZUMS.REC.1391.1456. Individuals with T2D were enrolled from the eye clinic and the diabetic outpatient clinic between December 2014 and November 2018. A control group of 114 healthy individuals was also obtained in the study as a secondary target with the goal of comparison. The inclusion criteria consisted of all type 2 diabetic patients between the ages of 30 and 70 years old. Patients with a known history of ocular, nephrological, or neurological disorders unrelated to diabetes mellitus, such as malignancies, infectious, vascular, or autoimmune diseases were excluded, along with the patients who were unwilling to continue with the study. A written informed consent was obtained from the participants. In the case of illiterate participants, the study was explained thoroughly and verbally before obtaining the informed consent. A total of 206 patients with type 2 diabetes were studied in this case-control study with a focus on diabetic microangiopathy. The control group consisted of patients with type $2 \mathrm{DM}$ without the respective diabetic microangiopathy.

The diagnosis of type 2 diabetes was based on the World Health Organization/American Diabetes Association description of diabetes [15]. Diabetic microangiopathy is specified as a small vessel disorder. The microangiopathies of attentiveness were categorized into diabetic retinopathy, nephropathy, and neuropathy, as previously described [16, 17]. These are summarized as follows: Medical examination: The presence of diabetic retinopathy was determined by dilated ophthalmic examination and fundus fluorescein angiography, which were performed by an expert ophthalmologist. Diabetic retinopathy was classified as non-proliferative diabetic retinopathy (NPDR) and proliferative diabetic retinopathy (PDR) [18].

Diabetic nephropathy was determined by assessing two separate 24-h urine collection samples. Nephropathy was classified as an albumin excretion rate of 30-300 $\mathrm{mg} / \mathrm{l} / 24 \mathrm{~h}$ as microalbuminuria and $>300 \mathrm{mg} / \mathrm{l} / 24 \mathrm{~h}$ as gross albuminuria in a timed urine collection after excluding urinary tract infection [19].

Clinical evaluation for peripheral neuropathy was carried out by neurophysiologic studies with nerve conduction velocity $(\mathrm{NCV})$. The number of nerves involvement of the (both median, ulnar, peroneal, posterior tibial motor nerves, and both median, ulnar, sural sensory nerves) determined the clinical staging of peripheral neuropathy as follows: stage 0 as no neuropathy; stage 1 as one or two nerves affected (mild); stage 2 as three or four nerves affected (moderate); stage 3 as five or more nerves affected (severe) [20].

Autonomic dysfunction is evaluated based on the heart rate and blood pressure responses to a variety of stimuli. The cardiac parasympathetic response was tested by immediate heart rate response to standing, (30th beat, 15th beat ratios) $<1.00$ was abnormal. Postural fall in blood pressure to standing more than $30 \mathrm{mmHg}$ indicate sympathetic cardiac damage [21].

\section{DNA sample preparation}

Peripheral blood samples were collected from the antecubital vein from all subjects into ethylenediaminetetraacetic acid (EDTA) and processed for GST genotyping. A fasting blood sample was collected with the purpose of determination of blood sugar (FBS) level, as well as the percentage of glycosylated hemoglobin (HbA1c) [22]. Genomic DNA was extracted using a Macrogen DNA purification kit (Macrogen Inc., South Korea). All kits and reagents were utilized according to their manufacturer's instructions.

\section{Genotyping of GSTT1 and GSTM1 polymorphism}

Genotypes for GSTT1 and GSTM1 were evaluated through multiplex polymerase chain reaction (PCR) methods [23]. This molecular analysis allows us to detect the present genotype (homozygote or heterozygote) or null genotype (homozygous complete deletion of both alleles). Human $\beta$-globin locus was used as an internal control to ensure the success of the amplification process, in the case of null genotypes for GSTM1 and 
GSTT1 loci. Details of all primers are shown in Table 1. The PCR results were confirmed by electrophoresis in a $2 \%$ agarose gel and visualized using the SYBR Safe dye. Gel electrophoresis of DNA from samples positive for GSTM1 and GSTT1 genotypes showed bands of 240 and $480 \mathrm{bp}$, respectively, while the control Albumin PCR product was attributed to $315 \mathrm{bp}$ (Fig. 1).

\section{Statistical analyses}

Statistical analysis was performed using SPSS version 18.0. GSTT1 and GSTM1 genotypes were divided into two categories of null (homozygous deletion) or nondeleted. The relationship between GSTT1 and GSTM1 genotypes and microangiopathy of T2 DM was assessed by the means of the odds ratio (OR) analysis with 95\% confidence intervals (CI). Departure in the distribution of genotypes from Hardy-Weinberg equilibrium was assessed through the chi-square test. $P$ value $<0.05$ was considered statistically significant.

\section{Results}

A dataset of two hundred-six T2D patients and 114 diabetes-free participants were included in this study. Mean age, sex, body mass index, duration of diabetes, serum $\mathrm{HbA1c}$ level, history of hypertension, and hyperlipidemia of the examined subjects and a part of patients with diabetic microangiopathy in our study group are summarized in Table 2. Analysis of the distribution by age and sex proved no significant differences between the study groups; therefore, homogeneity between groups was observed. After adjustment regarding age and sex, a relationship between the occurrence of diabetic microangiopathy and higher levels of HbA1c and longer duration of diabetes was observed. Data analyses revealed that the significance of the effect of the duration of diabetes of non-microangiopathy versus microangiopathy diabetic patients was less than 0.001. In addition, the magnitude of the effect of elevated HbA1c on non-microangiopathy versus microangiopathy diabetic patients was less than 0.001 (Table 2).

A total of 109 (52.91\%) patients with microangiopathy suffered from retinopathy, 57 (27.67\%) from nephropathy, and 110 (53.4\%) from neuropathy. Among the patients with diabetic nephropathy, $64.9 \%$ were categorized into microalbuminuria and $35.1 \%$ into gross

Table 1 PCR primers for GSTT1, GSTM1 loci

\begin{tabular}{ll}
\hline GSTM1-F & 5'-GAA CTC CCT GAA AAG CTA AAG C-3' \\
GSTM1-R & 5'-GTT GGG CTC AAA TAT ACG GTG G-3' \\
GSTT1-F & 5'-TC CTT ACT GGT CCT CAC ATC TC-3' \\
GSTT1-R & $5^{\prime}$-TCA CCG GAT CAT GGC CAG CA-3' \\
$\beta$-globin-F & 5'-GAA GAG CCA AGGACA GGT AC-3' \\
3-globin-R & $5^{\prime}$-CAA CTT CATCCA CGT TCA CC-3' \\
\hline
\end{tabular}

albuminuria. In patients with diabetes, peripheral neuropathy was observed in 102 subjects $(49.51 \%)$ who were categorized as follows: $57.8 \%$ mild, $28.4 \%$ moderate, and $13.7 \%$ severe neurophysiologic changes. Whereas autonomic dysfunction was observed in 60 subjects $(29.13 \%)$ divided as $16.7 \%$ sympathetic, $50 \%$ parasympathetic, and $33.3 \%$ both categories of autonomic neuropathy. One hundred thirty-four (65\%) of diabetic patients had at least one form of microangiopathy.

Among the control group, the frequency of GSTM1 null and GSTT1 null was $13.16 \%$ and $21.05 \%$ respectively, while in T2D patients the frequency of GSTM1 null and GSTT1 null was $9.7 \%$ and $23.79 \%$ respectively. These distributions were in accordance with the HardyWeinberg equilibrium. The frequencies of null GSTM1 and null GSTT1 genotypes were $5.56 \%$ and $16.67 \%$, respectively, among the uncomplicated DM patients. The frequencies of null GSTM1 and GSTT1 genotype in the microvascular complicated DM group were 11.94\% (16/ $134)$ and $27.61 \%(37 / 134)$, respectively. Table 3 demonstrates the distribution of the frequency of GSTM1 genotypes and their relation to clinical status in both T2D patients and controls in details.

We observed an association between the null genotype of GSTM1 with a higher risk of developing microvascular renal complications of T2D. The proportion of GSTM1 null genotype was higher in diabetic nephropathy compared to non-nephropathy $(19.3 \%$ vs. $6.04 \%, P=$ 0.006).

Regarding GSTT1 locus, more susceptibility to diabetic peripheral neuropathy was associated with gene deletion compared to that of diabetic patients without peripheral neuropathy $(30.39 \%$ vs. $17.31 \%)(P=0.02)$. Additionally, a statistically marginally significant association was observed between the GSTT1 null genotype and the occurrence of microvascular complications in T2D patients $(P=0.054)$ The relation between GSTT1 deletion and risk of microangiopathy can be observed in Table 4 in detail.

However, the rate of microvascular complications in patients with diabetes did not differ significantly between different GSTM1 genotype groups. In addition, no statistically significant differences were found between the control and T2D groups regarding the deletion in GSTM1 or GSTT1 genes.

\section{Discussion}

Increased production of reactive oxygen species and reduction in antioxidant defense mechanisms occurred as a result of DM. Oxidative stress has been considered as an important risk factor in the occurrences of diabetes and its complications [24, 25]. Many studies have dealt with GST polymorphism in various diseases, but only a few studies have investigated the role of GST 


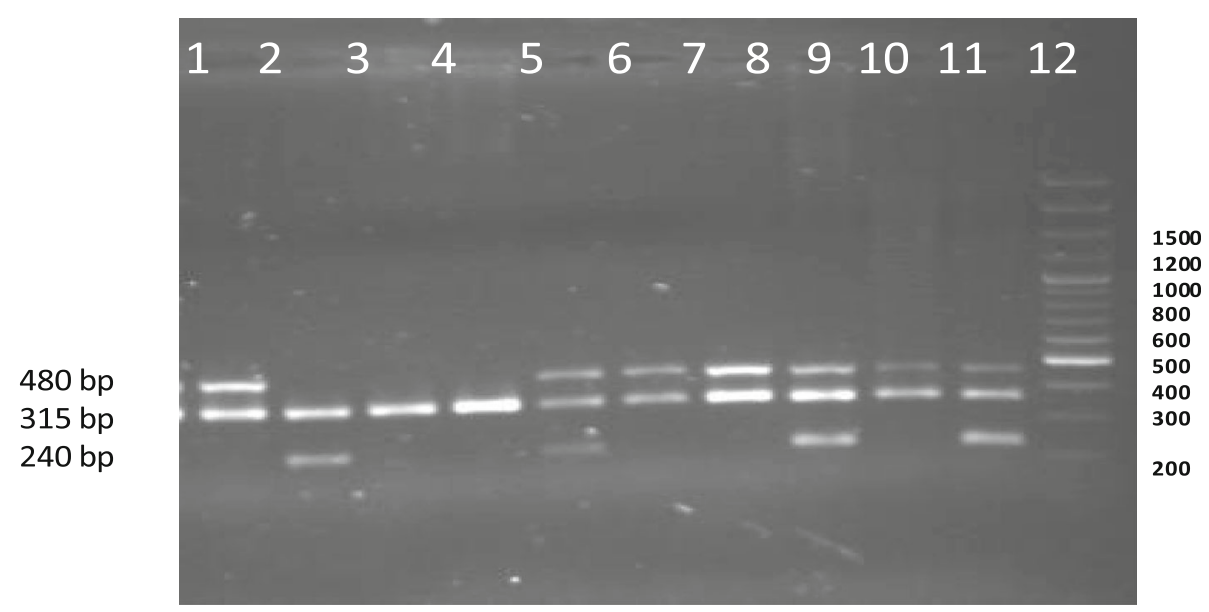

Fig. 1 A multiplex-PCR analysis of GSTM1 and GSTT1 gene polymorphism. GSTM1 and GSTT1 PCR products were analyzed directly by electrophoresis on a 1\% agaros gel. GST11(480 bp), GSTM1 (240 bp) and Albumin (315 bp) genes. Lane 3,4 GSM1-null/GSTT1-null, Lane 1,6,7,10 GSTM1-null/GSTT1-present, Lane 5,9,11 GSTM1-present/GSTT1-present, Lane 2 GSTM1-present/GSTT1-null sample. Lane 12 is a 50-bp DNA ladder

polymorphisms in diabetes and its complications [2632]. This molecular epidemiologic case-controlled study revealed that a lack of GSTM1 did not have any effect on susceptibility toward diabetic microangiopathy in general. Along with that, the occurrence of T2D was not affected by the presence of GSTM1/GSTT1 null genotype. However, the influence of this polymorphism on important clinical parameters related to some forms of diabetic microangiopathy was verified.

Fujita et al. found that GSTM1 null genotype was present in $48.6 \%$ of Japanese type 2 diabetic patients with nephropathy, versus $55.1 \%$ of patients without nephropathy. Based on their studies, the development of diabetic nephropathy was not affected by the presence of GSTM1 null genotype [33]. According to Sharma et al. reduced GSH plasma levels were found to have significant association with increased risk of renal damage in T2D [34]. A study conducted within the Brazilian population on the relationship between the genetic polymorphism of GSTM1, GSTT1, and GSTP1 in the risk of developing DN did not find any statistically significant association between the GSTM1, GSTT1, and GSTP1 null genotypes and the development of DN [35]. In contrast, in an Iranian study, null GSTT1 and GSTM1 genotypes increased the risk for end-stage renal disease by 1.8 times $(P<0.001)$ [36].

In our study, the GSTM1-null genotype conferred a statistically significant increased risk of diabetic nephropathy regarding the GSTM1-wild genotype $(\mathrm{OR}=3.72,95 \% \mathrm{CI}=1.451-9.523)$. This indicated that deletion in the GSTM1 gene placed the patient at higher risk for diabetic nephropathy. Downregulation of other antioxidant enzymes in the absence of the GSTM1 gene may also explain for its positive role in diabetic nephropathy [37].

A study investigating the expression of glutathione Stransferases class theta 1 (GSTT1) in type 2 DR subjects reported that highly significant differences $(P \leq 0.05)$ were observed in GSST1 expression in DR patients compared to diabetic and control groups [38].

Table 2 Clinical characteristics of the study population

\begin{tabular}{|c|c|c|c|c|}
\hline Variables & Control (114) & DM with microangiopathy (134) & DM without microangiopathy (72) & $P$ value \\
\hline Age (years) & $59.16 \pm 13.21$ & $56.97 \pm 10.46$ & $55.69 \pm 8.49$ & 0.376 \\
\hline $\operatorname{Sex}(M / F)$ & $45 / 69$ & $42 / 92$ & $21 / 51$ & 0.46 \\
\hline BMI $\left(\mathrm{kg} / \mathrm{m}^{2}\right)$ & $25.7 \pm 2.5$ & $25.9 \pm 2.7$ & $25.5 \pm 2.4$ & 0.642 \\
\hline Diabetes duration & N/A & $15.20 \pm 5.37$ & $11.26 \pm 5.32$ & $<0.001$ \\
\hline $\mathrm{HbA} 1 \mathrm{c}$ & N/A & $8.87 \pm 1.09$ & $7.18 \pm 0.52$ & $<0.001$ \\
\hline BP (systolic) & $138 \pm 13$ & $139 \pm 12$ & $141 \pm 13$ & 0.376 \\
\hline BP (diastolic) & $82 \pm 7$ & $83 \pm 6$ & $84 \pm 7$ & 0.224 \\
\hline TC (mg/dl) & $201 \pm 93$ & $218 \pm 69$ & $196 \pm 91$ & 0.116 \\
\hline TG $(\mathrm{mg} / \mathrm{dl})$ & $215 \pm 88$ & $222 \pm 87$ & $201 \pm 87$ & 0.239 \\
\hline
\end{tabular}

$B M I$ body mass index; $B P$ blood pressure; DM diabetes mellitus; $H b A 1 c$ hemoglobin A1c; $M$ male; F female; TC total cholesterol; TG triglyceride Data are expressed as mean \pm standard deviation (SD) or $n(\%)$ 
Table 3 The relationship between GSTM1 genotypes and microangiopathic complications in diabetic patients

\begin{tabular}{llll}
\hline Groups/variables & Null & Wild & $P$ value \\
\hline DM without DR vs. & $13 / 97(13.4)$ & $84 / 97(86.6)$ & 0.073 \\
DM with DR & $7 / 109(6.42)$ & $102 / 109(93.58)$ & $140 / 149(93.96)$ \\
DM without DN vs. & $9 / 149(6.04)$ & $46 / 57(80.7)$ & 0.006 \\
DM with DN & $11 / 57(19.3)$ & $95 / 104(91.35)$ \\
DM without DNp vs. & $9 / 104(8.65)$ & $91 / 102(89.22)$ \\
DM with DNp & $11 / 102(10.78)$ & $133 / 146(91.1)$ \\
DM without DNa vs. & $13 / 146(8.9)$ & $53 / 60(88.33)$ \\
DM with DNa & $7 / 60(11.67)$ & $68 / 72(94.44)$ \\
DM without microangiopathy vs. & $4 / 72(5.56)$ & $118 / 134(88.06)$ & 0.389 \\
DM with microangiopathy & $16 / 134(11.94)$ & $99 / 114(86.84)$ & 0.354 \\
Control vs. & $15 / 114(13.16)$ & $186 / 206(90.3)$ & 0.107 \\
DM & $20 / 206(9.7)$ & 0.222 \\
\hline
\end{tabular}

$D M$ diabetes mellitus; $D R$ diabetic retinopathy; $D N$ diabetic nephropathy; $D N p$ peripheral diabetic neuropathy; $D N a$ autonomic diabetic neuropathy The data are expressed as in $n$ (\%)

Our study did not confirm an association between GSTM1 gene polymorphism and other microangiopathic lesions in our diabetic patients. This is inconsistent with the observations of other studies that demonstrated a positive association between GSTM1-null genotype and diabetic retinopathy, which was reported in some of these studies on type 1 diabetes [39-41]. In contrast, Cilenšek et al. found that GSTM1 null genotype had a protective role against diabetic retinopathy $(\mathrm{OR}=0.475$, $95 \% \mathrm{CI}=0.339-0.668$ ), while the GSTT1 null genotype was associated with an increased risk of diabetic retinopathy $(\mathrm{OR}=2.303,95 \% \mathrm{CI}=1.649-3.216)$ in $\mathrm{T} 2 \mathrm{D}$ [42]

In our study, the GSTT1-null genotype was found to be significantly more frequent in the cases of diabetic neuropathy compared to those of non-neuropathic patients $(\mathrm{OR}=2.087,95 \% \mathrm{CI}=1.077-4.032)$ emphasizing the role GSTT1-null genotype to predisposing the patient to a higher risk for diabetic neuropathy in subjects with T2D.

Doney et al. showed that the GSTT1 genotype was associated with the progression of both nephropathy and diabetic retinopathy in subjects with T2D [43]. Stoian et al. showed that the GSTM1/GSTT1 null genotype was associated with neither the risk of developing T2D nor the occurrence of diabetic neuropathy [44]. Dadbinpour et al. showed that the GSTT1 null genotype was not associated with the presence of DR in T2D patients $(P=0.187)$ but noticed an association between GSTM1 genotype and diabetic retinopathy $(P=0.04)$, in opposition with a Slovenian study which revealed that GSTM1 null genotype might have protective effects against diabetic retinopathy [40]. Moasser et al. showed that lack of GSTM1 and the presence of GSTM1/GSTT1 null genotypes together were associated with the development T2D but there was no significant effect on the occurrence of DR [35]. A Slovakian study reported that GSTM1 and GSTT1 gene polymorphisms were associated with an increased risk of cardiovascular autonomic neuropathy in adolescents with T1D [45].

In the meta-analysis with 25 studies, the data revealed that the GSTT1 null $(\mathrm{OR}=1.56 ; 95 \% \mathrm{CI}=1.38-1.77)$ and combined GSTM1-GSTT1 null genotypes (OR = $1.91 ; 95 \% \mathrm{CI}=1.25-2.94)$ increased the risk for development of T2D-related complications, but not the GSTM1

Table 4 The relationship between GSTT1 genotypes and microangiopathic complications in diabetic patients

\begin{tabular}{llll}
\hline Groups/variables & Null & Wild & $P$ value \\
\hline DM without DR vs. & $18 / 97(18.56)$ & $79 / 97(81.44)$ & 0.104 \\
DM with DR & $31 / 109(28.44)$ & $78 / 109(71.56)$ & $114 / 149(76.51)$ \\
DM without DN vs. & $35 / 149(23.49)$ & $43 / 57(75.44)$ & 0.857 \\
DM with DN & $14 / 57(24.56)$ & $86 / 104(82.69)$ \\
DM without DNp vs. & $18 / 104(17.31)$ & $71 / 102(69.61)$ \\
DM with DNp & $31 / 102(30.39)$ & $112 / 146(76.71)$ \\
DM without DNa vs. & $34 / 146(23.29)$ & $45 / 60(75)$ & 0.02 \\
DM with DNa & $15 / 60(25)$ & $60 / 72(83.33)$ \\
DM without microangiopathy vs. & $12 / 72(16.67)$ & $97 / 134(72.39)$ \\
DM with microangiopathy & $37 / 134(27.61)$ & $90 / 114(78.95)$ & 0.462 \\
Control vs. & $24 / 114(21.05)$ & $157 / 206(76.21)$ & 0.054 \\
DM & $49 / 206(23.79)$ & 0.34 \\
\hline
\end{tabular}

$D M$ diabetes mellitus; $D R$ diabetic retinopathy; $D N$ diabetic nephropathy; $D N p$ peripheral diabetic neuropathy; $D N a$ autonomic diabetic neuropathy The data is demonstrated in $n(\%)$ 
null genotype [46]. Results of recently reported studies assessed the association between GST and T2D and its complications according to Preferred Reporting Items for Systematic reviews and Meta-Analyses demonstrated separate or combination of GSTM1 and GSTT1 null genotypes were associated with T2D. In addition, GSTM1 and combination of GSTM1/GSTT1 null genotypes were also associated with $\mathrm{DN}$ [47].

Heterogeneity may result in the conflicting result obtained from genetic studies on the influence of GST genes polymorphisms on microvascular complications of T2D which requires further investigation to elucidate the role of these polymorphisms in the development of diabetic microvascular complications.

The studies that evaluate the association and impact of genotype on disease progression are usually limited by the fact that more chronic complications will develop with longer follow-up. Considering that the duration of diabetes is an important risk factor for microangiopathic complications, our results must be interpreted with caution.

We speculate that mutant detoxification enzymes such as GSTs may result in the induction of other GST transferases and antioxidant proteins, which represent compensation to increase in the intracellular levels of antioxidative products.

Our study presents some limitations. Firstly, the restricted areas of patients' selection with a limited period of follow-up are major limitations that may cause the obtained results to be inconclusive for the general population. Further researches in different ethnic groups in large scale and cohort studies are suggested. Another limitation of our study was the lack of direct biochemical evidence demonstrating the correlation of GSTM1 and GSTT1 mutations with altered catalytic activities against their substrates. Such evidence may be confirmed by utilizing cDNA construction and transfection into incultured cell media. Furthermore, other genetic polymorphisms involved in ROS production/elimination such as manganese superoxide dismutase (MnSOD), catalase (CAT), and glutathione-peroxidase-1(GPX1) were not evaluated, which are recommended to be studied for future projects. However, despite such limitations, it still may be a valuable investigation of GSTM1/ GSTT1, and their effect on microvascular complications in T2D. Our study results thus represent only a part of the intricate pathobiologic matrix of diabetic microangiopathy.

The fact that prevalence and severity of microangiopathic complications increase with poor glycemic and longer diabetes duration is undeniable, however, it is true in a general view and not in individual level. Several studies have shown the impact of gene polymorphisms and have suggested the influence of other factors.
Although we categorized microangiopathic complications of patients according to the severity of diseases, no significant relations were found. This may be in part related to a limited number of cases. More studies would require detailed information on the gene-gene and geneenvironmental interactions.

\section{Conclusion}

In conclusion, we found a statistically significant association between GSTM1-null genotype and susceptibility to diabetic nephropathy. The GSTT1-null genotype may also contribute to the development of diabetic peripheral neuropathy in T2D, along with a marginal significance regarding the GSTT1 deletion and the occurrence of microvascular complications of T2D. We hope that our study will contribute to better understanding the pathways leading to diabetes complications and to help better identify and manage these microvascular complications especially in individuals at higher genetic susceptibility.

\section{Abbreviations}

T2D: Type 2 diabetes; DM: Diabetes mellitus; DR: Diabetic retinopathy; DN: Diabetic nephropathy; DNp: Peripheral diabetic neuropathy;

DNa: Autonomic diabetic neuropathy

\section{Acknowledgements \\ The authors would like to thank the "Clinical Research Development Unit" at Bu-Ali Sina Hospital, Mazandaran Universty of Medical Sciences, Sari, Iran dearly for their assistance.}

\section{Presentation at a meeting}

This article has not been presented at any meeting or conference. It has not been submitted for publication at any other journal simultaneously.

\section{Authors' contributions}

MBHS contributed to the conception and design of the study, and also interpretation of the data. AAA contributed to the analysis and interpretation of data as well as drafting and revision of the manuscript. MRSR contributed to the design of the study as well as data acquisition. AAA contributed to data analysis and interpretation as well as the final draft and revision of the manuscript. RA contributed to the conception and design of the study as well as data acquisition. AAA contributed to the conception and design of the study, data acquisition, analysis, and interpretation, and manuscript draft and revision. FD contributed to the design of the study as well as data acquisition and interpretation and manuscript revision. All authors approve of the submitted version for publication. The authors agree to be personally accountable for the integrity and accuracy of the study.

\section{Funding}

This study was not financially granted by any organization.

\section{Availability of data and materials}

The datasets used and/or analyzed during the current study are available from the corresponding author on reasonable request.

\section{Ethics approval and consent to participate}

This study was approved by Mazandaran University of Medical Sciences Ethics Committee on 24 September 2014 under approval ID: IR.MAZUMS.REC.1391.1456. A written informed consent was obtained from the participants. In the case of illiterate participants, the study was explained thoroughly and verbally before obtaining the informed consent. 


\section{Competing interests}

The authors declare that they have no competing interests.

\begin{abstract}
Author details
${ }^{1}$ Molecular and Cell Biology Research Center, Mazandaran University of Medical Science, Sari, Iran. ${ }^{2}$ Department of Medicine, Tehran University of Medical Sciences, Tehran, Iran. ${ }^{3}$ Department of Ophthalmology, Bu-Ali Sina Hospital, Mazandaran University of Medical Sciences, Sari, Iran. ${ }^{4}$ Faculty of Medicine, Mazandaran University of Medical Sciences, Sari, Iran. ${ }^{5}$ Diabetes Research Center, Mazandaran University of Medical Sciences, Sari, Iran. ${ }^{6}$ Department of Public Health, Mazandaran University of Medical Sciences, Sari, Iran.
\end{abstract}

Received: 2 March 2020 Accepted: 1 July 2020

Published online: 01 September 2020

\section{References}

1. American Diabetes Association. Introduction: standards of medical care in diabetes - 2018. Diabetes Care 2018; 41(Supplement 1):S1-2. https://doi.org/ 10.2337/dc18-Sint01

2. Rizvi S, Raza ST, Mahdi F (2014) Association of genetic variants with diabetic nephropathy. World J Diabetes 5(6):809-816

3. Pitocco D, Tesauro M, Alessandro R, Ghirlanda G, Cardillo C (2013) Oxidative stress in diabetes: implications for vascular and other complications. Int J Mol Sci 14(11):21525-21550

4. Dandona P, Aljada A (2002) A rational approach to pathogenesis and treatment of type 2 diabetes mellitus, insulin resistance, inflammation, and atherosclerosis. Am J Cardiol 90:27G-33G

5. Bayness JW, Thorpe SR (1999) Role of oxidative stress in diabetic complications (a new perspective to an old paradigm). Diabetes 48:1-9

6. Hayes JD, Strange RC (1995) Potential contribution of the glutathione Stransferase supergene family to resistance to oxidative stress. Free Radic Res 22:193-207

7. Pinheiro DS, Rocha Filho CR, Mundim CA, de Marco JP, Ulhoa CJ, Reis AS et al (2013) Evaluation of glutathione S-Transferase GSTM1 and GSTT1 deletion polymorphisms on type-2 diabetes mellitus risk. PLoS ONE 8(10):15

8. Rinaldi R, Eliasson E, Swedmark S, Morgenstern R (2002) Reactive intermediates and the dynamics of glutathione transferases. Drug Metab Dispos 30:1053-1058

9. Garte S (2001) Metabolic susceptibility genes as cancer risk factors: time for a reassessment? Cancer Epidemiol Biomark Prev 10:1233-1237

10. Rebbeck TR (1997) Molecular epidemiology of the human glutathione Stransferase genotypes GSTM1 and GSTT1 in cancer susceptibility. Cancer Epidemiol Biomark Prev 6:733-743

11. Cotton SC, Sharp L, Little J, Brockton N (2000) Glutathione S transferase polymorphisms and colorectal cancer. Am J Epidemiol 151:7-32

12. Coggan M, Whitbread L, Whittington A, Board P (1998) Structure and organization of the human theta-class glutathione S-transferase and Ddopachrome tautomerase gene complex. Biochem J 334:617-23. 10

13. Zaki MA, Moghazy TF, El-Deeb MMK, et al. Glutatione S-transferase M1, T1 and $\mathrm{P} 1$ gene polymorphisms and the risk of developing type 2 diabetes mellitus in Egyptian diabetic patients with and without diabetic vascular complications. Alex J Med. 2014 https://doi.org/10.1016/j.ajme.2014.03.003.

14. Purkait P, Halder K, Roy AG, Sarkar BN, Naidu JM (2014) GSTM1 null genotype associated with type 2 diabetic nephropathy patients among Indian population. World J Pharm Res 3:4452-4463

15. Expert Committee on the Diagnosis and Classification of Diabetes Mellitus: Report of the expert committee on the diagnosis and classification of diabetes mellitus. Diabetes Care. 2003;26(Suppl. 1):S5-S20

16. Adeghate E, Saadi H, Adem A, Obineche E. Diabetes mellitus and its complications: molecular mechanisms, epidemiology, and clinical medicine, Ann NY Acad Sci. 2006;1084(1)

17. Ahmadzadeh Amiri A, Hashemi-Soteh MB, Haghshenas MR, Daneshvar F, Rastegar A, Farazmand T (2013) Haptoglobin polymorphism in individuals with type 2 diabetic microangiopathy. North Am J Med Sci 5:529-535

18. Diabetes Control and Complications Trial Research Group, Nathan DM et al (1993) The effect of intensive treatment of diabetes on the development and progression of long-term complications in insulin-dependent diabetes mellitus. N Engl J Med 329(14):977-986
19. Cheung CK, Swaminathan R (1987) Rapid, economical immunoturbidimetric method for microalbuminuria. Clin Chem 33:204

20. Bao XH, Wong V, Wang Q, Low LC (1999) Prevalence of peripheral neuropathy with insulin-dependent diabetic mellitus. Pediatr Neunol 20: 204-209

21. Ewing DJ, Clarke BF (1982) Diagnosis and management of diabetic autonomic neuropathy. Br Med J 285:916-918

22. Maquart FX, Gillery P, Bernard JF, Mante JP, Borel JP (1980) A method for specially measuring of hemoglobin $\mathrm{A} 1 \mathrm{C}$ with a disposable commercial ionexchange column. Clin Chim Acta 108:329-332

23. Chen CL, Liu Q, Relling MV (1996) Simultaneous characterization of glutathione S-transferase M1 and T1 polymorphisms by polymerase chain reaction in American whites and blacks. Pharmacogenetics. 6:187-191

24. $\mathrm{Ha} \mathrm{H}$, Kim KH (1995) Role of oxidative stress in the development of diabetic nephropathy. Kidney Int 48(Suppl. 51):S18-S21

25. Lehmann R, Schleicher ED (2000) Molecular mechanisms of diabetic nephropathy. Clin Chim Acta 297:135-144

26. Spurdle AB, Chang JH, Byrnes GB, Chen X, Dite GS, McCredie MR et al (2007) A systematic approach to analyzing gene-gene interactions: polymorphisms at the microsomal epoxide hydrolase EPHX and glutathione S-transferase GSTM1, GSTT1, and GSTP1 loci and breast cancer risk. Cancer Epidemiol Biomark Prev 16:769-774

27. Abu-Amero KK, Al-Boudari OM, Mohamed GH, Dzimiri N (2006) T null and M null genotypes of the glutathione S-transferase gene are risk factor for CAD independent of smoking. BMC Med Genet 7:38

28. Miller EA, Pankow JS, Millikan RC, Bray MS, Ballantyne CM, Bell DA et al (2003) Glutathione-S-transferase genotypes, smoking, and their association with markers of inflammation, hemostasis, and endothelial function: the atherosclerosis risk in communities (ARIC) study. Atherosclerosis. 171(2):265-272

29. Hayek T, Stephens JW, Hubbart CS, Acharya J, Caslake MJ, Hawe E et al (2006) A common variant in the glutathione $S$ transferase gene is associated with elevated markers of inflammation and lipid peroxidation in subjects with diabetes mellitus. Atherosclerosis. 184(2):404-412

30. Burim RV, Canalle R, Martinelli ADLC, Takahashi CS (2004) Polymorphisms in glutathione S-transferases GSTM1, GSTT1 and GSTP1 and cytochromes P450 CYP2E1 and CYP1A1 and susceptibility to cirrhosis or pancreatitis in alcoholics. Mutagenesis. 19:291-298

31. Colombo J, Rossit ARB, Caetano A, Borim AA, Wornrath D, Silva AE (2004) GSTT1, GSTM1 and CYP2E1 genetic polymorphisms in gastric cancer and chronic gastritis in a Brazilian population. World J Gastroenterol 10:1240-1245

32. D'Alo F, Voso MT, Guidi F, Massini G, Scardocci A, Sica S et al (2004) Polymorphisms of CYP1A1 and glutathione S-transferase and susceptibility to adult acute myeloid leukemia. Haematologica. 89:664-670

33. Fujita $H$, Narita $T$, Meguro $H$, Shimotomai T, Kitazato H, Kagaya E et al (2000) No association of glutathione S-transferase M1 gene polymorphism with diabetic nephropathy in Japanese type 2 diabetic patients. Nephrology. 22: 479-486

34. Sharma M, Gupta S, Singh K, Mehndiratta M, Gautam A, Kalra OP et al (OctDec 2016) Association of glutathione-S-transferase with patients of type 2 diabetes mellitus with and without nephropathy. Diabetes Metab Syndr 10(4):194-197

35. de Lima RM, dos Anjosa LRB, Alves TB, Coelho ASG, Pedrino GR, Santosa RS, et al. Do GST polymorphisms influence in the pathogenesis of diabetic nephropathy? Molecular and Cellular Endocrinology Volume 478, 15 December 2018, Pages 10-16.

36. Nomani H, Hagh-Nazari L, Aidy A, Vaisi-Raygani A, Kiani A, Rahimi Z (2016 Oct) Association between GSTM1, GSTT1, and GSTP1 variants and the risk of end stage renal disease. Ren Fail 38(9):1455-1461

37. Otto-Knapp R, Jurgovsky K, Schierhorn K, Kunkel G (2003) Antioxidative enzymes in human nasal mucosa after exposure to ozone: possible role of GSTM1 deficiency. Inflamm Res 52:51-55

38. Sharif S, Maqsood M, Naz S, Manzoor F, Shah Jahan M, Farasat T (2019) Expression of Gstt1 in type 2 diabetic retinopathy patients. Crit Rev Eukaryot Gene Expr 29(1):47-50

39. Hovnik T, Dolzan V, Bratina NU, Podkrajsek KT, Battelino T (2009) Genetic polymorphisms in genes encoding antioxidant enzymes are associated with diabetic retinopathy in type 1 diabetes. Diabetes Care 32:2258-2262

40. Dadbinpour A, Sheikhha MH, Darbouy M, Afkhami-Ardekani M (2013) Investigating GSTT1 and GSTM1 null genotype as the risk factor of diabetes type 2 retinopathy. J Diabetes Metab Disord 12:48 
41. Moasser E, Azarpira N, Shirazi B, Saadat M, Geramizadeh B (2014) Genetic polymorphisms of glutathione-s-transferase M1 and T1 genes with risk of diabetic retinopathy in Iranian population. Iran J Basic Med Sci 17:351-356

42. Cilenšek I, Mankoč S, Petrovič MG, Petrovič D (2012) GSTT1 null genotype is a risk factor for diabetic retinopathy in Caucasians with type 2 diabetes, whereas GSTM1 null genotype might confer protection against retinopathy. Dis Markers 32:93-99

43. Doney AS, Lee S, Leese GP, Morris AD, Palmer CN (2005) Increased cardiovascular morbidity and mortality in type 2 diabetes is associated with the glutathione $\mathrm{S}$ transferase theta-null genotype. A Go-DARTS study. Circulation. 111:2927-2934

44. Stoian A, Banescu C, Bslasa RI, Moususianu A, Stoian M, Moldovan VG, et al. Influence of GSTM1, GSTT1, and GSTP1 polymorphisms on type 2 diabetes mellitus and diabetic sensorimotor peripheral neuropathy risk. Dis Markers. 2015: 638693. DOl: https://doi.org/10.1155/2015/638693

45. Vojtkova J, Durdik P, Ciljakova M, Michnova Z, Turcan T, Babusikova E (2013) The association between glutathione S-transferase $\mathrm{T} 1$ and $\mathrm{M} 1$ gene polymorphisms and cardiovascular autonomic neuropathy in Slovak adolescents with type1diabetes mellitus. J Diabetes Complicat 27:44-48

46. Nath S, Das S, Bhowmik A, Ghosh SK, Choudhury Y . The GSTM1 and GSTT1 null genotypes increase the risk for type 2 diabetes mellitus and the subsequent development of diabetic complications: a meta-analysis. Curr Diabetes Rev 2019;15(1):31-43.

47. Bitarafan F, Khodaeian M, Tabatabaei-Malazy O, Amoli MM (2019 Sep) Influence of antioxidants' gene variants on risk of diabetes mellitus and its complications: a systematic review. Minerva Endocrinol 44(3):310-325

\section{Publisher's Note}

Springer Nature remains neutral with regard to jurisdictional claims in published maps and institutional affiliations.

\section{Submit your manuscript to a SpringerOpen ${ }^{\circ}$ journal and benefit from:}

- Convenient online submission

- Rigorous peer review

- Open access: articles freely available online

- High visibility within the field

- Retaining the copyright to your article

Submit your next manuscript at $\boldsymbol{\nabla}$ springeropen.com 